\title{
The structure of liquid carbon dioxide and carbon disulfide
}

J. Neuefeind, H. E. Fischer, J. M. Simonson, A. Idrissi, A. Schöps, and V. Honkimäki

Citation: J. Chem. Phys. 130, 174503 (2009); doi: 10.1063/1.3116106

View online: https://doi.org/10.1063/1.3116106

View Table of Contents: http://aip.scitation.org/toc/jcp/130/17

Published by the American Institute of Physics

\section{Articles you may be interested in}

An optimized molecular potential for carbon dioxide

The Journal of Chemical Physics 122, 214507 (2005); 10.1063/1.1924700

Structure and dynamics of liquid $\mathrm{CS}_{2}$ : Going from ambient to elevated pressure conditions

The Journal of Chemical Physics 145, 154505 (2016); 10.1063/1.4964816

Molecular dynamics study of liquid carbon disulfide and benzene: Effect of pressure on the far-infrared collisioninduced absorption

The Journal of Chemical Physics 103, 9580 (1995); 10.1063/1.469973

A general purpose model for the condensed phases of water: TIP4P/2005

The Journal of Chemical Physics 123, 234505 (2005); 10.1063/1.2121687

Comparison of simple potential functions for simulating liquid water

The Journal of Chemical Physics 79, 926 (1983); 10.1063/1.445869

A smooth particle mesh Ewald method

The Journal of Chemical Physics 103, 8577 (1995); 10.1063/1.470117

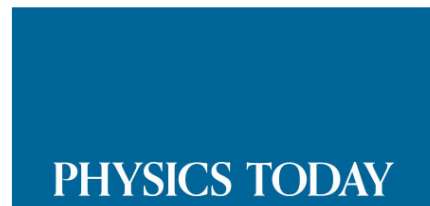




\title{
The structure of liquid carbon dioxide and carbon disulfide
}

\author{
J. Neuefeind, ${ }^{1, a)}$ H. E. Fischer, ${ }^{2}$ J. M. Simonson, ${ }^{3}$ A. Idrissi, ${ }^{4}$ A. Schöps, ${ }^{5}$ and \\ V. Honkimäki ${ }^{6}$ \\ ${ }^{1}$ Spallation Neutron Source, Oak Ridge National Laboratory, 1 Bethel Valley Rd., \\ Oak Ridge, Tennessee 37831, USA \\ ${ }^{2}$ Institut Laue-Langevin, 6 rue Jules Horowitz, BP 156, 38042 Grenoble cedex 9, France \\ ${ }^{3}$ Center for Nanophase Materials Sciences, Oak Ridge National Laboratory, P.O. Box 2008 MS 6493, \\ Oak Ridge, Tennessee 37831, USA \\ ${ }^{4}$ Laboratoire de Spectrochimie Infrarouge et Raman Université des Sciences et Technologies de Lille, \\ 59655 Villeneuve d'Ascq, France \\ ${ }^{5}$ Hamburger Synchrotronstrahlungslaboratorium (HASYLAB), Deutsches Elektronensynchrotron (DESY), \\ Notkestr 85, 22603 Hamburg, Germany \\ ${ }^{6}$ European Synchrotron Radiation Facility (ESRF), BP 220, 38043 Grenoble Cedex 9, France
}

(Received 29 September 2008; accepted 19 March 2009; published online 1 May 2009)

\begin{abstract}
We present neutron and x-ray scattering data (a $2 N+X$ experiment) of liquid $\mathrm{CO}_{2}$ and $\mathrm{CS}_{2}$ at a density of about 10 molecules $/ \mathrm{nm}^{3}$. Because the scattering length contrast of the carbon isotope is very small and, in fact, smaller than anticipated from standard scattering length tables, a direct partial structure factor determination via matrix inversion gives unconvincing results. Instead we search for the best representation of the three independent scattering data sets by a simulation of rigid molecules interacting via a 12-6-1 potential, furthermore restricting the pressure $p$, the density $\rho$, and the temperature $T$ to the experimental values. We show that a 12-6-1 potential is completely adequate to describe the structure of $\mathrm{CO}_{2}$; for $\mathrm{CS}_{2}$ we find that the best 12-6-1 potential still slightly overestimates the height of the sulfur-sulfur pair-distribution function $g_{\mathrm{SS}}$. Orientational correlations reflect the similarities much more than the differences of the two molecular systems. The distinct differences in the atom-atom pair distribution functions of $\mathrm{CO}_{2}$ and $\mathrm{CS}_{2}$ do not mean that their structures are radically different and the comparison with the crystalline structures is somewhat deceptive. A linear transformation, wherein all the parameters describing the interaction and the geometry of $\mathrm{CS}_{2}$ are changed to those of $\mathrm{CO}_{2}$, allows us to point out the physical parameters which may be responsible for the differences or similarities in thermodynamic behavior (pressure) and structures (orientations) between the two liquids. (C) 2009 American Institute of Physics.
\end{abstract} [DOI: $10.1063 / 1.3116106$ ]

\section{INTRODUCTION}

Why would anyone want to redetermine the structure of liquid carbon dioxide and liquid carbon disulfide? It is hard to argue that $\mathrm{CO}_{2}$ is not the molecule on everybody's mind: being considered at the same time a "green" solvent, ${ }^{1}$ but also the most prominent of the "greenhouse" gases assumed to be responsible for increasing global temperatures. ${ }^{2,3}$ However, in fact, both $\mathrm{CO}_{2}$ and $\mathrm{CS}_{2}$ have been investigated extensively by neutron and x-ray diffraction (Refs. 4-6, and references therein), going back more than 30 years ${ }^{7}$ complemented by both classical (Refs. 8-10, and references therein) and Car-Parinello-type simulations. ${ }^{11,12}$ None of these earlier studies have used more than one scattering experiment, however, except for the interesting approach in Ref. 13 that combines the neutron data of Ref. 7 and the x-ray data of Ref. 14. This means, previous scattering experiments have been limited to determine total structure factors that are a weighted average of the element-specific partial structure factor information. Improved experimental techniques have made partial structure determinations possible for systems such as

\footnotetext{
${ }^{a)}$ Author to whom correspondence should be addressed. FAX: (+1) 865574 6080. Electronic mail: zjn@sns.gov.
}

$\mathrm{GeO}_{2},{ }^{15} \mathrm{HF}^{16}$ and $\mathrm{ZnCl}_{2}$ (Ref. 17) that previously would have been impossible or overly difficult. We will present the first determination of partial structure factors of the $\mathrm{CO}_{2}$ and $\mathrm{CS}_{2}$ by a combination of a ${ }^{12} \mathrm{C} /{ }^{13} \mathrm{C}$ neutron isotope substitution experiment with an $\mathrm{x}$-ray scattering experiment, also called a $2 N+X$ (Ref. 18) experiment.

Many earlier (total) structure determinations of $\mathrm{CO}_{2}$ have focused on determining its structure over a wide range of densities (e.g., Refs. 4, 19, and 20). In this work we will concentrate instead on how different or similar the structures of $\mathrm{CO}_{2}$ and $\mathrm{CS}_{2}$ are at the same molecular density. At room temperature equal molecular density is achieved if $\mathrm{CO}_{2}$ is at a pressure of about 66 bars and $\mathrm{CS}_{2}$ at ambient pressure. To expand on the point of comparing these two similar yet distinct molecules we ask naively why it is that $\mathrm{CS}_{2}$ is a liquid at ambient conditions and $\mathrm{CO}_{2}$ a gas? $\mathrm{CS}_{2}$ is certainly the heavier molecule and a popular ${ }^{21}$ yet flawed argument explains the periodic trends of increasing melting and boiling points of the group 6 hydrides $\mathrm{H}_{2} \mathrm{O}, \mathrm{H}_{2} \mathrm{~S}, \mathrm{H}_{2} \mathrm{Se}, \mathrm{H}_{2} \mathrm{Te}$, and $\mathrm{H}_{2} \mathrm{Po}$ with increasing mass, $\mathrm{H}_{2} \mathrm{O}$ standing out because of its hydrogen bonding. Ignoring small structural isotope effects, ${ }^{22}$ however, the static properties such as the structure do not depend on the molecular mass. ${ }^{23}$ If an ultraheavy 


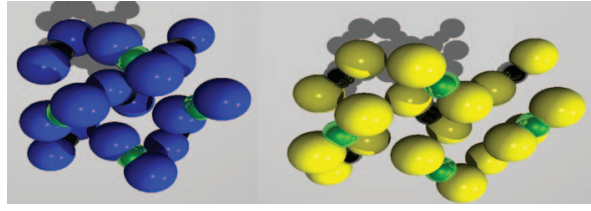

FIG. 1. (Color) Crystal structures of $\mathrm{CO}_{2}-\mathrm{I}$ (left) and $\mathrm{CS}_{2}$ (right) seen along the $a$-axis. $\mathrm{C}$ atoms are shown in black (background) and green (foreground), $\mathrm{O}$ in blue, and $\mathrm{S}$ in yellow.

oxygen isotope ${ }^{32} \mathrm{O}$ would exist with the same mass as $\mathrm{S}$, $\mathrm{C}^{32} \mathrm{O}_{2}$ and $\mathrm{H}_{2}{ }^{32} \mathrm{O}$ would still be structurally and thermodynamically a $\mathrm{CO}_{2}$ and an $\mathrm{H}_{2} \mathrm{O}$ and not a $\mathrm{CS}_{2}$ and an $\mathrm{H}_{2} \mathrm{~S}$. The mass therefore cannot be the reason why $\mathrm{CS}_{2}$ is a liquid and $\mathrm{CO}_{2}$ is not at ambient conditions. Furthermore, the physical size of the molecules- $\mathrm{CS}_{2}$ being larger than $\mathrm{CO}_{2}$-would actually suggest the opposite behavior, namely, that higher pressure rather than lower pressure would be required to maintain $\mathrm{CS}_{2}$ at the same density as $\mathrm{CO}_{2}$. Therefore we expect differences in the interaction potential rather that the mass or the size of the molecule to be responsible for its different behavior.

Furthermore, $\mathrm{CO}_{2}$ forms a number of crystalline phases at high pressure that have attracted a lot of recent attention, ${ }^{24,25}$ but at normal pressure it forms a simple cubic pyrite-type structure, ${ }^{26} \mathrm{CS}_{2}$ forms an orthorhombic structure. ${ }^{27}$ The molecular axes of all next neighbors in solid $\mathrm{CO}_{2}$ are perpendicular to each other. Looking at the crystal structures of both compounds in comparison (Fig. 1) suggests that parallel orientations may be a more important structure element in $\mathrm{CS}_{2}$ than it is in $\mathrm{CO}_{2}$.

In order to have complete experimental structures of $\mathrm{CO}_{2}$ and $\mathrm{CS}_{2}$, we planned to use a $2 N+X$ experiment involving the carbon isotope pair to determine the partial structure factors of $\mathrm{CO}_{2}$ and $\mathrm{CS}_{2}$. Unfortunately, in the course of this study it was found that the scattering contrast between the carbon isotopes is very small, substantially smaller yet than standard table values would suggest. ${ }^{28}$ That makes the direct matrix inversion of the equation system used to determine the partial structure factors ill defined (see below). We rely on molecular dynamics simulations of molecules interacting through Lennard-Jones and electrostatic total potential models, seeking the optimal parameters leading to the best representation of the three independent scattering data sets. Indeed, modeling techniques such as reverse Monte Carlo ${ }^{29}$ and empirical potential structure refinement ${ }^{30}$ (EPSR) have been used to obtain a solution indirectly, a solution constrained by the requirement of obtaining a three-dimensional (3D) arrangement of molecules of the correct (=experimental) density. It seems to be a logic extension to seek a 3D arrangement of molecules that not only correctly represents the experimental density but also the correct pressure and temperature at that density. We have therefore chosen to take a different approach which constrains the solution with the thermodynamic state variables of the system for a fixed functional form of the atomic potentials. Even though introducing a new methodology is not the main purpose of this paper it is expected that the method developed in the process may be applicable beyond the context of this particu- lar system and it is described in some detail in Sec IV. This allows us to get the best parameters of the potential models used. Finally, we perform a series of simulations in which we change the parameters from those characteristic of $\mathrm{CS}_{2}$ to those characteristic of $\mathrm{CO}_{2}$, in order to isolate physical parameters responsible for the differences in the thermodynamic behavior between the two systems.

\section{EXPERIMENT}

The neutron diffraction measurements were all carried out on the D4c instrument at the Institut Laue-Langevin ${ }^{31}$ using the $0.5 \AA$ wavelength setup; high energy (140 and 89 $\mathrm{keV}$, respectively) $\mathrm{x}$-ray diffraction measurements were made at the BW5 beamline at HASYLAB $\left(\mathrm{CO}_{2}\right.$ data) (Ref. 32) and at the ID15b beamline at the ESRF $\left(\mathrm{CS}_{2}\right.$ data). ${ }^{33}$ All measurements were carried out at ambient temperature. For the $\mathrm{CO}_{2}$ an active temperature control was implemented and the temperature was held at $298.1 \mathrm{~K}$; the $\mathrm{CS}_{2}$ data were measured at the ambient temperature of the reactor $(300.4 \mathrm{~K})$ that was found to be constant within a couple of tenths of a degree. The molecular density of both systems was held within $0.3 \%$ of $0.00993 \AA^{-3}$, occurring at atmospheric pressure for $\mathrm{CS}_{2}$ and at $\sim 66$ bars for $\mathrm{CO}_{2}$. The $\mathrm{CO}_{2}$ was enclosed in an aluminum pressure cell described earlier ${ }^{34}$ that allows investigation of samples with $\mathrm{x}$ rays and neutrons in the same sample environment in order to minimize systematic errors. This capability is especially useful in this case as it is necessary to reproduce the same thermodynamic state in both neutron and the x-ray experiments as precisely as possible, especially since $\mathrm{CO}_{2}$ is fairly close to its critical point under the experimental conditions, and therefore small changes in pressure and temperature lead to fairly large changes in the density. For neutron scattering, pressure cells made from TiZr alloy are more commonly used-an alloy in which Ti with negative scattering length and $\mathrm{Zr}$ with positive scattering length is randomly distributed onto the lattice sites and therefore does not produce Bragg powder lines. However, TiZr is still a quite strong scatterer and it does show structured diffuse scattering similar to the sample signal. Furthermore TiZr is fairly inconvenient material for $\mathrm{x}$-ray pressure cells: The trick that leads to the cancellation of Bragg powder lines in the neutron case does not apply and there are many of them, since the material is hexagonal ${ }^{35}$ rather than cubic as is aluminum. The $\mathrm{CS}_{2}$ sample was contained in a (conventional) thin walled $\mathrm{V}$ cell that has a vanishing coherent cross section in the neutron case and in glass capillaries for the $\mathrm{x}$-ray experiment. ${ }^{13} \mathrm{C}$ enriched samples were purchased from Cambridge Isotopes. The ${ }^{12} \mathrm{C}$ sample of $\mathrm{CO}_{2}$ is a sample enriched in ${ }^{12} \mathrm{C}$; the $\mathrm{CS}_{2}$ sample contains the natural C isotope mix with $98.9 \%{ }^{12} \mathrm{C}$, called ${ }^{12} \mathrm{CS}_{2}$ for simplicity in the following. The isotopic composition of the $\mathrm{CO}_{2}$ samples was checked by mass spectrometry and found to agree with specifications.

The usual scans of the empty diffractometer, the empty cell, and the sample in the cell were carried out, as well as a V-bar for normalization in the neutron case. Because the $\mathrm{CO}_{2}$ cell is made out of Al, it does show powder lines (Fig. 2). Unfortunately, due to strain buildup the scattering of the cell 

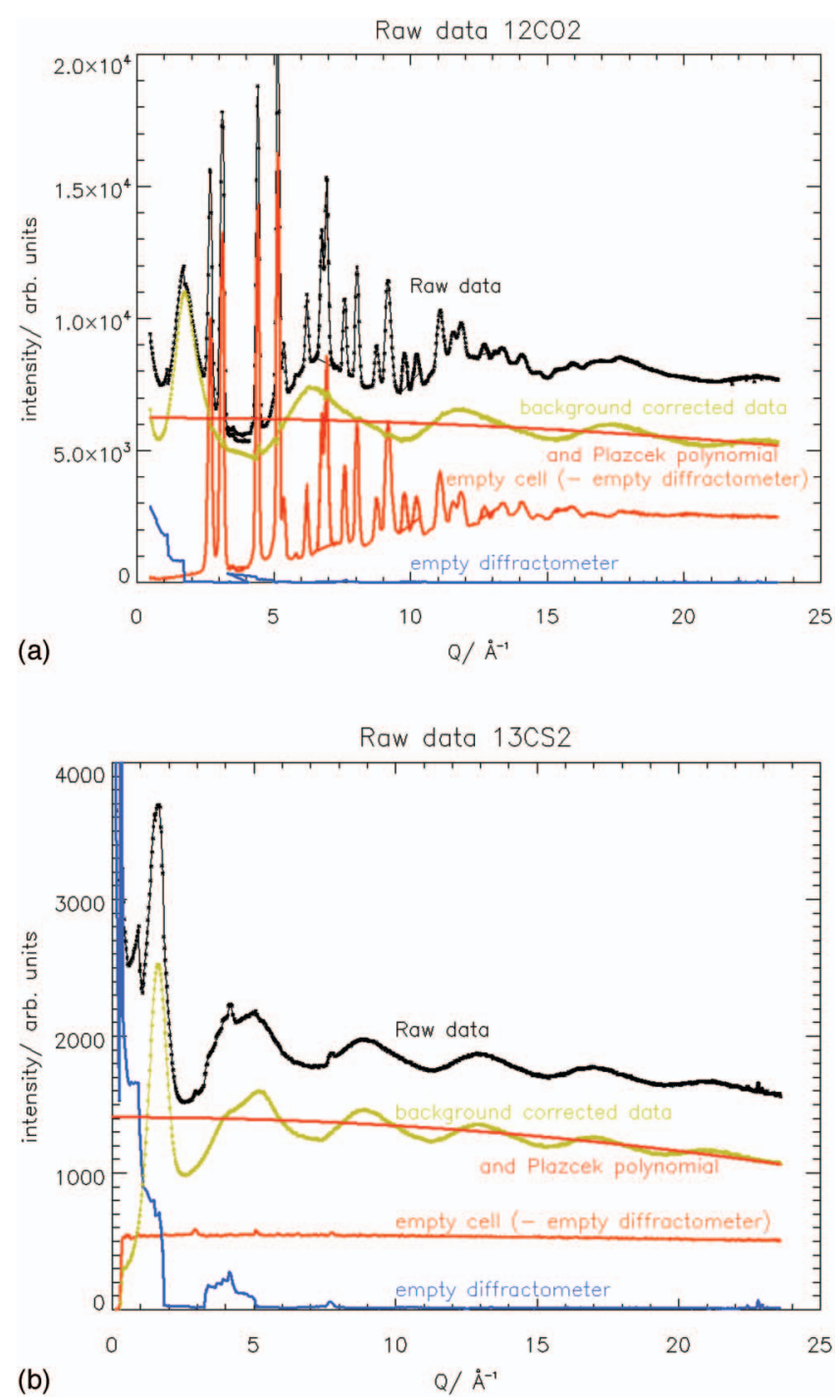

FIG. 2. (Color) Raw data for the ${ }^{13} \mathrm{CO}_{2}$ (a) and ${ }^{12} \mathrm{CS}_{2}$ (b) neutron scattering experiments as an example (as annotated).

without sample and with sample under pressure inside is not exactly the same. Therefore an additional measurement was carried out with the cell filled with Ar (that scatters neutrons very little) at the same pressure and temperature. Still the background subtraction in the region close to the Bragg lines is not perfect. Those data points were therefore excluded from further analysis.

Furthermore a small $(\sim 375 \mathrm{ppm})$ contamination of the ${ }^{13} \mathrm{CS}_{2}$ with a hydrogen-containing substance was detected by the presence of an incoherent scattering background. This small $\mathrm{H}$ contamination is also detectable by the presence of a $Q^{4}$ term in the Plazcek polynomial. Extensive discussion of this point has been presented in Ref. 28. Since the coherent cross section of hydrogen is smaller by a factor of about 40 than the incoherent cross section the impact of the $\mathrm{H}$ impurity on the coherent signal is negligible. However, as we want to compare equal to equal in an isotope substitution experiment, an additional measurement of a ${ }^{12} \mathrm{CS}_{2}$ sample intentionally contaminated with a known amount of benzene has been carried out. This allows us to assess how the signal from the ${ }^{12} \mathrm{CS}_{2}$ data would have looked, would the same
H-contamination have been present. Alternatively, we can analyze the (uncontaminated) ${ }^{13} \mathrm{CS}_{2}$ sample at the total structure level, or even try to correct the ${ }^{13} \mathrm{CS}_{2}$ for its H-content. It will be shown that effect of this correction on our result is minimal as expected.

The X-ray measurements of the $\mathrm{CO}_{2}$ sample were carried out with a single point Ge solid-state detector, while the $\mathrm{CS}_{2}$ measurements were obtained with a MAR image plate area detector. The area detector measurements offer excellent statistical accuracy, however they usually have higher systematic inaccuracies, for example, caused by an energy dependent efficiency and little collimation. ${ }^{36}$ To optimize the statistical accuracy obtained from the single point detector, the $\mathrm{CO}_{2}$ data were collected in two scan ranges with longer counting times at low- $Q$ (where the scattering function shows more detail) and shorter counting times at high- $Q$ (which in this case is mainly used for normalization).

Since we will compare our data with a simulation that is based on a rigid molecule model, the intramolecular scattering contributions should be calculated and removed before the comparison as the simulation obviously does not reflect the flexibility of the real molecules. For this purpose, we have fitted and subtracted a Debye type contribution:

$$
\begin{aligned}
\operatorname{intra}\left(\frac{d \sigma}{d \Omega}\right)= & 4 b_{\mathrm{C}} b_{\mathrm{S} / \mathrm{O} j_{0}}\left(Q r_{\mathrm{C}(\mathrm{O} / \mathrm{S})}\right) \exp \left(-Q^{2} \gamma^{2} / 2\right) \\
& +2 b_{\mathrm{S} / \mathrm{O} j_{0}}^{2}\left(2 Q r_{\mathrm{C}(\mathrm{O} / \mathrm{S})}\right) \exp \left(-Q^{2} \gamma^{2}\right)
\end{aligned}
$$

The CS distance $r_{\mathrm{CS}}=1.554 \AA$ and the CO distance $r_{\mathrm{CO}}=1.156 \AA$ in good agreement to earlier determinations. Note that here the OO, SS distance is set to twice the CO, CS distance, implying a perfectly straight molecule. There is recent discussion based on simulation results ${ }^{11}$ whether the equilibrium bond angle in $\mathrm{CO}_{2}$ is different from $180^{\circ}$. In our opinion, all deviations from $2 r_{\mathrm{C}(\mathrm{O} / \mathrm{S})}=r_{(\mathrm{O} / \mathrm{S})(\mathrm{O} / \mathrm{S})}$ can be explained by the apparent shortening expected for a vibrating linear molecule: At any give moment in time, the bond angle in a vibrating linear molecule is smaller than $180^{\circ}$. In any case these deviations are small and not relevant for the determination of the intermolecular structure.

\section{DETERMINATION OF PARTIAL STRUCTURE FACTORS}

A scattering experiment always provides a total structure factor $S$ as a function of the momentum transfer $Q$, which is a weighted sum of the element-specific partial structure factors (PSFs) $s_{i j}$ :

$$
\begin{aligned}
S(Q)= & w_{\mathrm{CC}} s_{\mathrm{CC}}(Q)+w_{\mathrm{C}(\mathrm{O} / \mathrm{S})} s_{\mathrm{C}(\mathrm{O} / \mathrm{S})}(Q) \\
& +w_{(\mathrm{OO} / \mathrm{SS})} s_{(\mathrm{OO} / \mathrm{SS})}(Q),
\end{aligned}
$$

where the weighting factors $w_{i j}$ depend on the magnitude of the interaction of the photons or neutrons with the atoms. If the scattering properties of the atoms can be modified, element-specific information can be obtained. The corresponding pair distribution functions (PDFs) $g_{i j}$ are then related to the structure factors $s_{i j}$ by a Fourier-sine transform. 


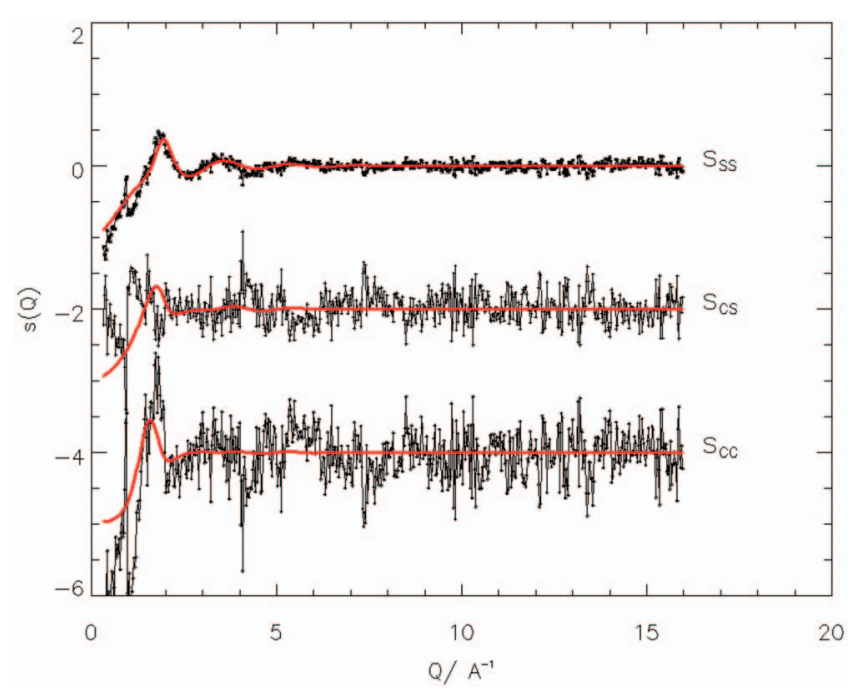

FIG. 3. (Color) Partial structure factors for $\mathrm{CS}_{2}$ obtained by direct matrix inversion. The experimental results are given with symbols joined by a line, the best model result (see below) is given for comparison with a solid red line, and $s_{\mathrm{CS}}$ and $s_{\mathrm{CC}}$ are shifted for clarity.

$$
r\left[g_{i j}(r)-1\right]=\frac{1}{2 \pi \rho} \int Q\left[s_{i j}(Q)-1\right] \sin (Q r) d Q
$$

Since Eq. (1) represents an equation system with three independent variables $s_{i j}$, three independent scattering experiments can determine the three PSFs; in our case these are two neutron experiments with the ${ }^{12} \mathrm{C}$ and ${ }^{13} \mathrm{C}$ variants of the molecules and an X-ray experiment. Equation (1) can therefore be written in matrix form

$$
\overline{\bar{w}} \cdot \bar{s}=\bar{S}
$$

Inversion of that equation system is formally:

$$
\bar{s}=\bar{S}(\overline{\bar{w}})^{-1} .
$$

However, if the information content in the three diffraction experiments is similar, inversion (4) becomes ill defined, which is the situation that we are faced with here. The directinversion solution via Eq. (4) for our $\mathrm{CS}_{2}$ data set is shown in Fig. 3 and the result is obviously not satisfying. Only the sulfur-sulfur partial structure factor driven by the contrast between the neutron and $\mathrm{x}$-ray experiments has a somewhat useful statistical and systematic accuracy. As the values of $\overline{\bar{w}}$ and hence $(\overline{\bar{w}})^{-1}$ are intrinsic properties of the system, there is little to be improved other than measuring $\bar{S}$ with the highest precision possible (and we would maintain that has been done). The only alternative left is to constrain the solution of Eq. (4). This is the approach taken here. Incorporating these constraints cannot be written down in the same concise way as Eq. (4) though and also the quality of the solution now obviously depends on the quality of the constraints. However, the $p, \rho$, and $T$ data of the systems are known with high precision and it is therefore expected that constraining the solution will actually improve it.

\section{SIMULATIONS}

Molecular dynamics simulations [MOSCITO (Ref. 37) code] were carried out all based on rigid molecule 12-6-1 type models, i.e., the potential $U$ contains Lennard-Jones interactions defined by the parameters $\varepsilon$ and $\sigma$ and chargecharge Coulombic interactions given by the partial charges $q$. The total potential thus writes

$$
\begin{aligned}
U & =\sum_{i \alpha} \sum_{j \beta}\left[U^{\mathrm{LJ}}\left(r_{i \alpha j \beta}\right)+U^{\mathrm{EL}}\left(r_{i \alpha j \beta}\right)\right] \\
& =\sum_{i \alpha} \sum_{j \beta}\left[4 \varepsilon_{i \alpha j \beta}\left(\left(\frac{\sigma_{i \alpha j \beta}}{r_{i \alpha j \beta}}\right)^{12}-\left(\frac{\sigma_{i \alpha j \beta}}{r_{i \alpha j \beta}}\right)^{6}\right)+\frac{q_{i \alpha} q_{j \beta}}{r_{i \alpha j \beta}}\right] .
\end{aligned}
$$

1000 molecules of $\mathrm{CO}_{2}$ and 2000 molecules of $\mathrm{CS}_{2}$, respectively, were brought in a cubic starting configuration and typically equilibrated for 10000 time steps of 5 fs duration. After that time $p, T$, and the energetic variables reached their equilibrium value, except for the softest $\mathrm{CO}_{2}$ potentials that required longer equilibration times. Then a production run of 50000 steps of $5 \mathrm{fs}$ is initiated. The simulations use a Verlettype neighborlist ${ }^{38}$ via a linkcell algorithm with a cutoff radius of $12 \AA$. A SHAKE ${ }^{39}$ procedure with a relative tolerance of $10^{-3}$ is used to restrict the molecular geometry. MOSCITO incorporates the usual minimum image conventions. The cutoff radius for all intermolecular potentials is $10 \AA$, with a long range correction of the charge interactions via a smooth particle-mesh Ewald summation. ${ }^{40}$

All the models are thus defined by six parameters, four $\mathrm{LJ}$ parameter $\varepsilon$ and $\sigma$ for $\mathrm{C}$ and $\mathrm{S} / \mathrm{O}$ (the mixed $\mathrm{LJ}$ parameters are given by Lorentz-Berthelot rules) the charges $q$ subject to the constraint of charge neutrality, and the CO/CS bond length. The charges and bond length are chosen to represent the experimental quadrupole moments of $\mathrm{CO}_{2}$ and $\mathrm{CS}_{2}\left(q_{C}=0.6512 e^{-}\right.$for $\mathrm{CO}_{2}$ and $q_{C}=-0.308 e^{-}$for $\left.\left.\mathrm{CS}_{2}\right)\right)^{41,42}$ The charges were not varied and also the $\varepsilon$ and $\sigma$ for the $\mathrm{C}$ are kept constant, because the shape of the molecule is largely determined by the $\mathrm{S} / \mathrm{O}$ atoms at the ends of the molecules. The model CS distance (from Ref. 42) is $1.56 \AA$, the CO distance (from Ref. 41) is $1.149 \AA$, compared to the 1.554 and $1.156 \AA$ that we found experimentally (see above), thus within a few thousands of an $\AA$. For technical reasons, the mass of the molecules is contained in two "virtual" sites on the molecular axis such as to maintain the total mass and the moment of inertia. ${ }^{43}$ To obtain the model that best represents the diffraction data we systematically varied $\varepsilon_{\mathrm{OO} / \mathrm{SS}}$, studying only pairs of $\left(\varepsilon_{\mathrm{OO} / \mathrm{SS}} \mid \sigma_{\mathrm{OO} / \mathrm{sS}}\right)$ that result in the correct pressure $p$ at the experimental $\rho, T$, thereby constraining our search by the experimental $p, \rho, T$ of the system that is known with high precision. Table I summarizes all parameter values.

By a series of test simulations we determine for each value of $\varepsilon_{\mathrm{OO} / \mathrm{SS}}$ the value of $\sigma_{\mathrm{OO} / \mathrm{SS}}$ for which the pressure is the experimental pressure (Fig. 4 left). Those values can be approximated (Fig. 4 right) by 
TABLE I. Potential parameter of the MD models.

\begin{tabular}{lcc}
\hline \hline & $\mathrm{CO}_{2}$ & $\mathrm{CS}_{2}$ \\
\hline$r_{\mathrm{C}(\mathrm{O} / \mathrm{S})} / \AA$ & 1.149 & 1.56 \\
Position virtual site/Å (from $\mathrm{C})$ & 0.9900 & 1.432 \\
mass virtual site/amu & 22.005 & 38.0705 \\
$\sigma_{\mathrm{C}} / \AA$ & 2.757 & 3.2 \\
$\varepsilon_{\mathrm{C}} / k_{B} \mathrm{~K}$ & 28.129 & 51.0 \\
$\sigma_{(\mathrm{O} / \mathrm{S})} / \AA$ & Variable & Variable \\
$\varepsilon_{(\mathrm{O} / \mathrm{S})} / k_{B} \mathrm{~K}$ & Variable & Variable \\
\hline \hline
\end{tabular}

$$
\sigma_{\mathrm{OO} / \mathrm{SS}}=A+B \varepsilon_{\mathrm{OO} / \mathrm{SS}} / k_{B}+C\left(\varepsilon_{\mathrm{OO} / \mathrm{SS}} / k_{B}\right)^{2} .
$$

For $\mathrm{CO}_{2}$ the values of the empirical constants $A, B$, and $C$ are $-4.957 \AA, 0.1589 \AA \mathrm{K}^{-1}$, and $-0.000756 \AA \mathrm{K}^{-2}$ within the range of $\varepsilon_{\mathrm{OO}}$ studied, that is between $70 \mathrm{~K}<\varepsilon_{\mathrm{OO}} / k_{B}$ $<100 \mathrm{~K}$. For $\mathrm{CS}_{2}$ the values of $A, B$, and $C$ are $2.426 \AA$, $0.00949 \AA \mathrm{K}^{-1}$, and $-1.8571 \times 10^{-5} \AA \mathrm{K}^{-2}$ in the range between $160 \mathrm{~K}<\varepsilon_{\text {SS }} / k_{B}<200 \mathrm{~K}$.

Thus, restricting $(p, \rho, T)$ to $(p, \rho, T)_{\exp }$ leaves us with just one independent parameter. Five simulations for each molecule within those ranges of $\varepsilon$ and with the corresponding values of $\sigma$ [i.e., for which $(p, \rho, T)=(p, \rho, T)_{\text {exp }}$ ] are then carried out to calculate the structure factors to be compared to the experiment. A polynomial of degree 2 was found sufficient to interpolate the structure factors for values of $\varepsilon / \sigma$ not simulated. Comparing the model to the experimental $S$
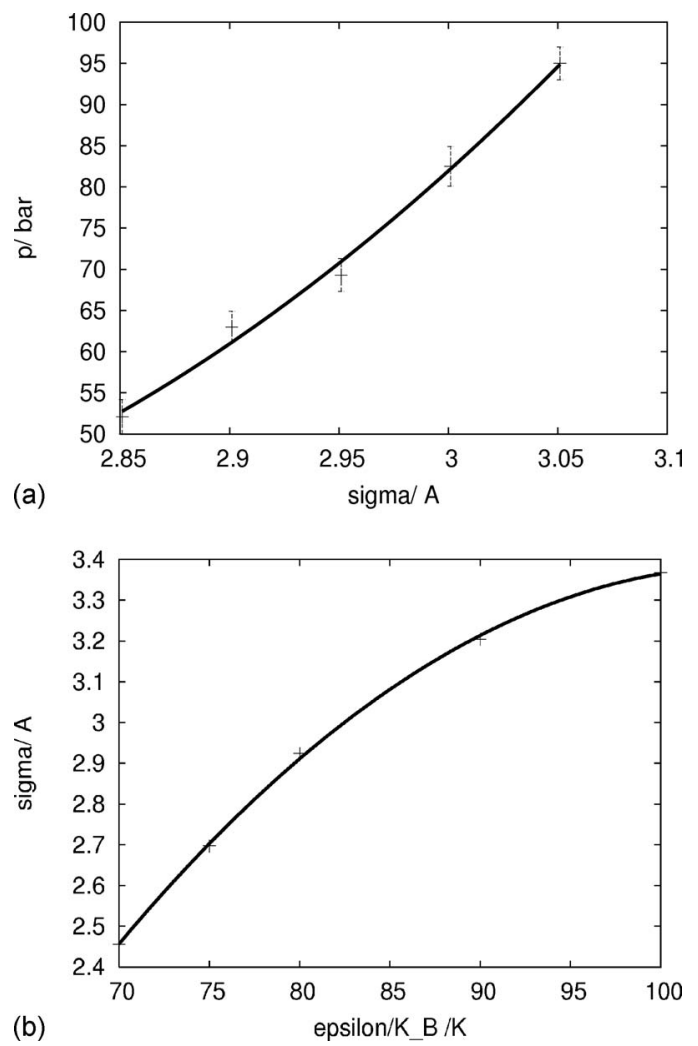

FIG. 4. a) Results for several test simulations with $\mathrm{CO}_{2}$ potentials with $\varepsilon_{\mathrm{OO}} / k_{B}=80 \mathrm{~K}$ and varying $\sigma_{\mathrm{OO}}$. Further test simulation were made at $\varepsilon_{\mathrm{OO}} / k_{B}=70,75,90$, and $100 \mathrm{~K}$. From these a $\sigma_{\mathrm{OO}}$ can be interpolated for which $(p, \rho, T)=(p, \rho, T)_{\exp }$ (again for $\left.\mathrm{CO}_{2}, \mathrm{~b}\right)$.

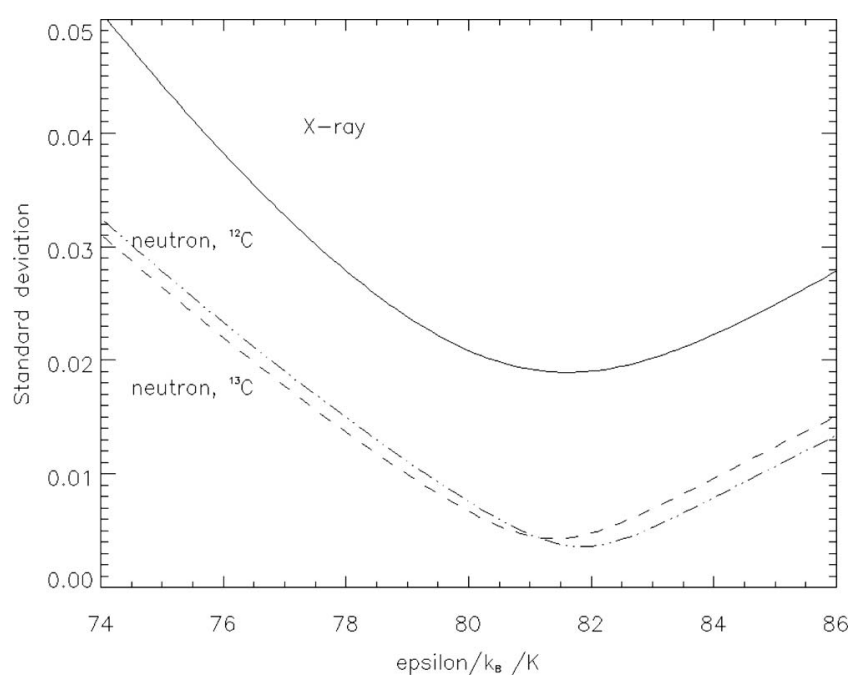

FIG. 5. Deviation of the model from the experimental total structure factors for $\mathrm{CO}_{2}$ as a function of the value of $\varepsilon_{\mathrm{OO}}$ for the three different experimental data sets, as annotated.

then leads to consistent, clearly defined minima in the standard deviations (Fig. 5) signaling the best model to describe the structure.

The model converges to the same $\varepsilon_{\mathrm{OO}}$ for $\mathrm{CO}_{2}$ for all three scattering experiment - that all have different weighting factors of the partials-within $0.2 \mathrm{~K}$ from $81.6 \mathrm{~K}$ in units of the Boltzmann constant $k_{B}$. That gives us high confidence that the partial PDFs of the model are indeed very close to the true partial PDFs of the system. The modeled and simulated total structure factors are compared in Fig. 6(a) and 6(b) (bottom). An increase in the scattering intensity at small $Q$ is visible both in simulation and experiment. It is caused by the proximity of the critical point and has been observed in previous experiments (e.g., Ref. 19) and has been reproduced in RMC modeling studies. ${ }^{44}$ The state point investigated here has a reduced density $\rho / \rho_{C}=1.56$ and is close but not very close to critical conditions and the small angle signal is therefore not very prominent. In the neutron case it is invisible unless a correction for the "umbrella effect" made, which causes the resolution of the instrument to deteriorate strongly at low $Q$.

For the $\mathrm{CS}_{2}$ case scale matching introduced an additional free parameter that scales the experimental data $(\sim 12 \%$ for the neutron- and $\sim 2 \%$ for the $\mathrm{x}$-ray data set). The derived models still have a larger variation in $\varepsilon$ than for the $\mathrm{CO}_{2}$ case. The result for the minimum varies from $\varepsilon_{S S} / k_{B}$ $=162.5 \mathrm{~K}$ for the $\mathrm{x}$-ray experiment to $176.8 \mathrm{~K}$ for the ${ }^{12} \mathrm{C}$ neutron experiment. The variation introduced by the correction for the $\mathrm{H}$-contamination discussed in the experimental section, however, is minute. The minimum for the ${ }^{12} \mathrm{C} \mathrm{S}_{2}$ neutron experiment shifts from $\varepsilon_{\mathrm{SS}} / k_{B}=176.8$ to $176.7 \mathrm{~K}$ (uncontaminated). The discrepancies discussed in the following therefore clearly have nothing to do with the presence of the $\mathrm{H}$-contamination in the ${ }^{13} \mathrm{C}$ sample. Comparison of the simulated and experimental PDFs shows that for all three experiments the model overestimates the density at $3.5 \AA$, the position of the main SS peak. That and the fact that $\varepsilon_{\mathrm{SS}}$ is lowest for the $\mathrm{x}$-ray case (which gives the highest weight to 

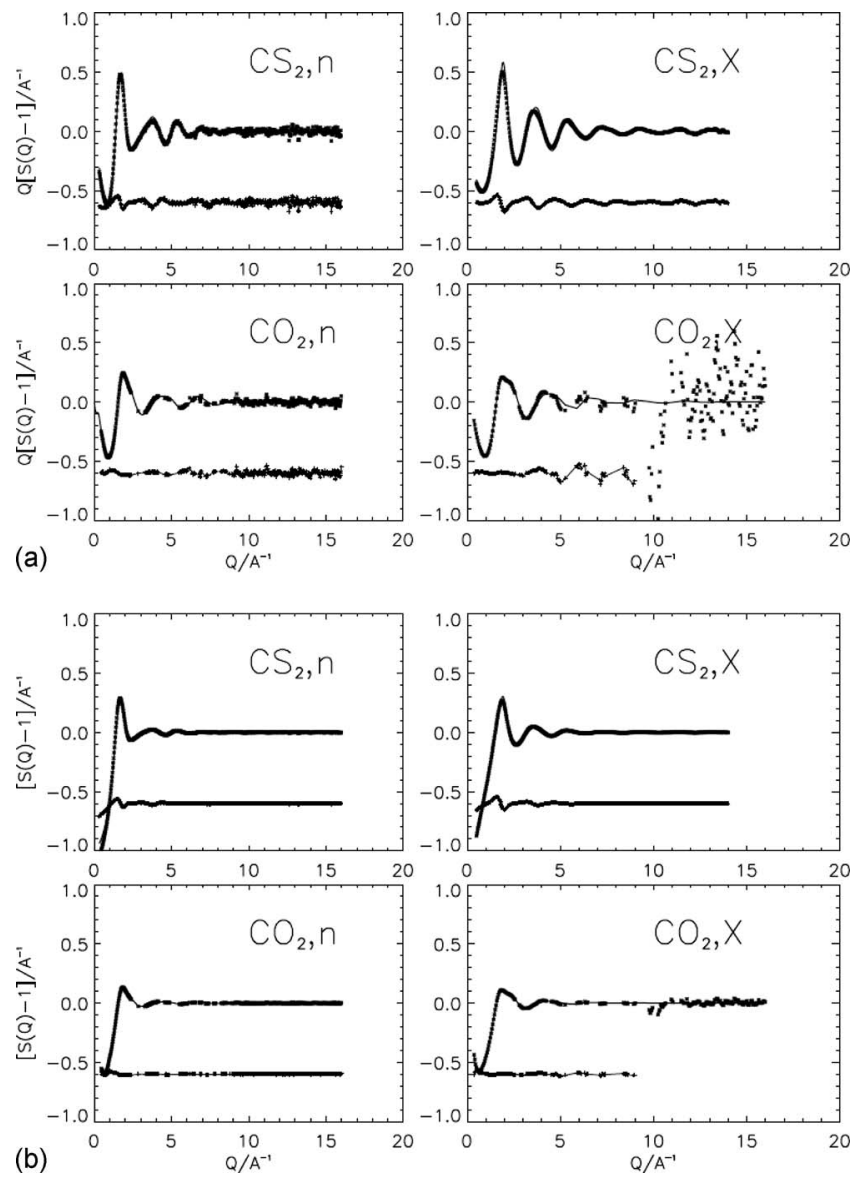

FIG. 6. (a) Modeled (line) and measured (symbols) $Q[S(Q)-1]$ and b) $[S(Q)-1]$. We show only the neutron measurements for the ${ }^{13} \mathrm{C}$ species and the $\mathrm{X}$-ray measurements, since the neutron measurements for the ${ }^{12} \mathrm{C}$ species are very similar to those for the ${ }^{13} \mathrm{C}$ species. Only the intermolecular part of the structure factor is shown. A residual is shown shifted by -0.6 units. The high- $Q$ portion of the data sets is truncated. The minimum $Q$ values are $0.45 \AA^{-1}\left(\mathrm{CO}_{2}-N\right), \quad 0.36 \AA^{-1}\left(\mathrm{CO}_{2}-X\right), \quad 0.33 \AA^{-1}\left(\mathrm{CS}_{2}-N\right), \quad$ and $0.50 \AA^{-1}\left(\mathrm{CS}_{2}-X\right)$

$s_{\mathrm{SS}}$ ) suggest that the real sulfur-sulfur correlation will be somewhat weaker than predicted by the models (on the order of $<10 \%$ ). The manifestation of that underestimation in $Q$-space is an oscillation in the residual that can be easily recognized in Figs. 6(a) and 6(b) (top).

It is noted that the situation that we encounter here is somewhat different than what is usually faced with EPSR simulations. While for EPSR usually a multitude of potentials is found that fits a given data set, ${ }^{30}$ here for $\mathrm{CS}_{2}$ we find none within the restrictions we impose that would fit all three data sets perfectly. That suggests however that it is possible to obtain a better fit to the data at the price of loosing the connection with the thermodynamics. It is possible to reduce the height of SS in the simulation by reducing $\varepsilon_{\mathrm{SS}}$, but at $(p, \rho, T)=(p, \rho, T)_{\exp }$ a reduced $\varepsilon_{\mathrm{SS}}$ implies a reduced $\sigma_{\mathrm{SS}}(\mathrm{cf}$. Fig. 4 right) and therefore position and height of the SS peak cannot be varied independently. It is not excluded that it is also possible to come up with more elaborate models that eventually might perfectly represent the scattering data and the thermodynamics. Such a more elaborate model could contain a different functional form for the potentials, polarizability, or charges that are not constrained to the atomic centers.

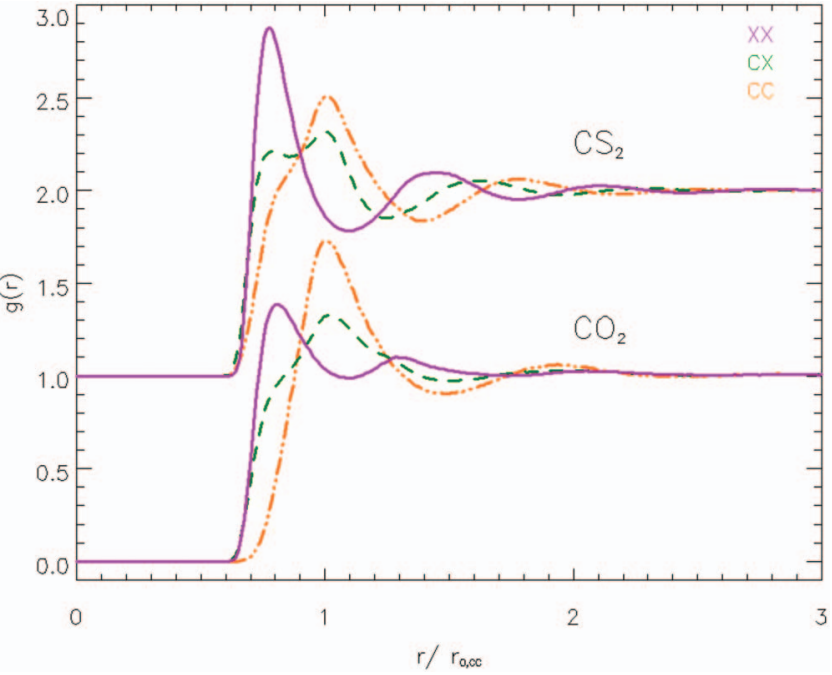

FIG. 7. (Color) Partial PDF $g_{\mathrm{OO} / \mathrm{SS}}$ (purple solid line), $g_{\mathrm{C}(\mathrm{O} / \mathrm{S})}$ (green broken line), and $g_{\mathrm{CC}}$ (orange line and dots) for $\mathrm{CS}_{2}$ (top) and $\mathrm{CO}_{2}$ (bottom). Note that the $r$-scale is normalized to the maximum of the $\mathrm{CC}$ pair distribution function. The $\mathrm{CS}_{2}$ data are shifted upward by one unit.

It is a legitimate question to ask if the additional restriction of structures to those that satisfy $(p, \rho, T)=(p, \rho, T)_{\exp }$ is useful if we are solely interested in structural properties. It is noted that by staying within the parameter space of simple 12-6-1 potentials, the widespread availability of MD programs such as Ref. 37 enables anyone to generate 3D models of $\mathrm{CO}_{2}$ and $\mathrm{CS}_{2}$ that are consistent with our diffraction data without having to have explicit access to our data. Thus the parameters of the 12-6-1 potential are a very concise way to convey the result of our experiments. For $\mathrm{CS}_{2}$ that statement has the qualifier that there is a residual discrepancy in the SS partial that cannot be resolved, possibly because the 12-6-1 is not a completely adequate description of the system, possibly because of remaining inaccuracies in the experiment. Beyond the aspect of convenience and conciseness of representation however it is felt, that the comparison of the deduced potential parameters furthers the understanding of the systems as shown in Sec. V.

\section{FURTHER RESULTS}

The resulting partial pair distribution functions for $\mathrm{CO}_{2}$ and $\mathrm{CS}_{2}$ presented in Fig. 7 are calculated for the average of the best-fit models, that is $\varepsilon_{\mathrm{OO}} / k_{B}=81.6 \mathrm{~K}, \sigma_{\mathrm{OO}}=2.969 \AA$ for $\mathrm{CO}_{2}$ and $\varepsilon_{\mathrm{SS}} / k_{B}=172.4 \mathrm{~K}, \sigma_{\mathrm{SS}}=3.509 \AA$ for $\mathrm{CS}_{2}$. The distance scale has been normalized to the peak position in the CC distribution function $\mathrm{r}_{0, \mathrm{CC}}$ at $3.95 \AA\left(\mathrm{CO}_{2}\right)$ and 4.95 $\AA\left(\mathrm{CS}_{2}\right)$. The most characteristic difference in the set of partial PDF is in $g_{\mathrm{OO} / \mathrm{SS}}$ : Both the first peak height and the position of the second peak change appreciably as we go from $\mathrm{CS}_{2}$ to $\mathrm{CO}_{2}$. That is still true if the peak height of $g_{\mathrm{SS}}$ is $10 \%$ smaller (see preceding paragraph). There are smaller changes in the low- $r$ shoulders to the $\mathrm{CO} / \mathrm{CS}$ and the $\mathrm{CC}$ partials as well.

In order to isolate, if possible, physical parameters responsible for the differences in thermodynamic behavior between the two liquids, we now perform a series of simulations in which we change the parameters of the model from 

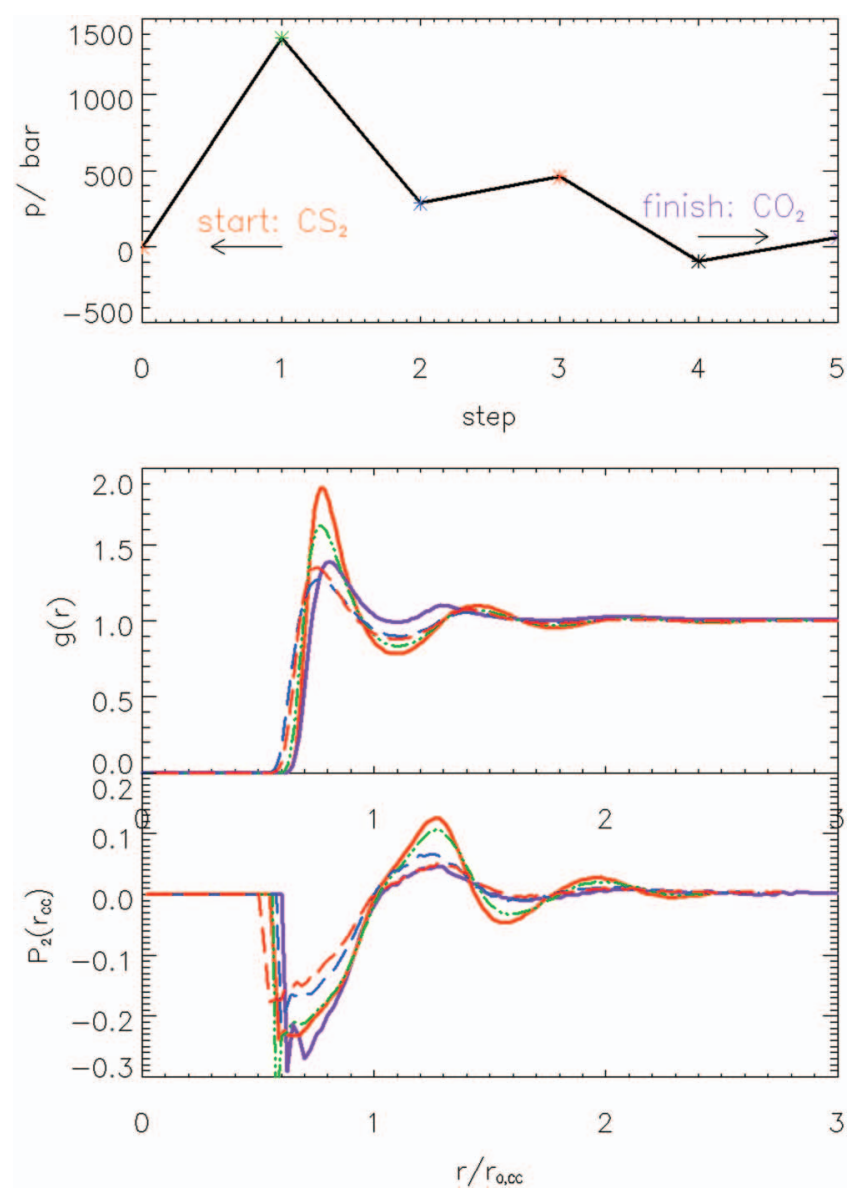

FIG. 8. (Color) The pressure (top), $g_{\text {oo/ss }}$ (middle) and $P_{2}[\cos (\theta)]$ (bottom) during the conversion of the potential parameters from $\mathrm{CS}_{2}$ to $\mathrm{CO}_{2}$. The changes made in each step are detailed in the main text, the colors in all three plots match. Step 0 is a solid line, step 1 line, two dots, step 2 broken line, step 3 broken line (very close), step five solid line.

those characteristic for $\mathrm{CS}_{2}$ to those characteristic for $\mathrm{CO}_{2}$. Four intermediate steps have been taken on the way from $\mathrm{CS}_{2}$ to $\mathrm{CO}_{2}$ : (1) $\varepsilon_{\mathrm{SS}}$ is changed to $\varepsilon_{\mathrm{OO}}$ (everything else is constant), (2) $\sigma_{\mathrm{SS} \rightarrow \mathrm{OO}}\left(\right.$ at $\left.\varepsilon=\varepsilon_{\mathrm{OO}}\right)$, (3) the carbon $\varepsilon_{\mathrm{CC}}$ and $\sigma_{\mathrm{CC}}$ are converted to the $\mathrm{CO}_{2}$ values, (4) the charges $q$ are reversed. In the final step (5) the bond length and the molecular mass is adjusted to make the conversion to $\mathrm{CO}_{2} \mathrm{com}$ plete. In order to characterize the molecular orientations, the second-order Legendre polynomial of the angle between the molecules $\Theta$ (Ref. 42) is calculated as a function of the CC distance:

$$
P_{2}[\cos (\theta)]=\left\langle\frac{3}{2} \cos ^{2}(\theta)-\frac{1}{2}\right\rangle,
$$

Figure 8 shows the pressure for each step along the conversion as well as the $g_{\mathrm{OO} / \mathrm{sS}}$ and $P_{2}$ defined above. The $\Delta p$ for each step along the conversion is larger than the net $\Delta p$. A " $\mathrm{CS}_{2}$ " molecule with the same size as $\mathrm{CS}_{2}$ but the small $\varepsilon_{\mathrm{OO}}$ of $\mathrm{CO}_{2}$ would indeed need a considerably higher $p$ to maintain at the same $\rho$ as explained in Sec. I. It should be noted that the order in which the parameters are converted is to some extend arbitrary; however, what is important to notice here that the net $\Delta p$ is the result of multiple competing effects each of which is larger than the actual experimental $\Delta p$ between $\mathrm{CO}_{2}$ and $\mathrm{CS}_{2}$. Furthermore, despite the sizable differences in $g_{\mathrm{OO} / \mathrm{sS}}$, the molecular orientations of $\mathrm{CO}_{2}$ and $\mathrm{CS}_{2}$ and all the hypothetical "in-between"-molecules are very similar-except for step 4 (an unstable $p<0$ configuration): The $P_{2}$ are negative (=preferentially parallel) on the leading edge of $g_{\mathrm{CC}}$, i.e., $r / r_{0, \mathrm{CC}}<1$, and positive (=preferentially perpendicular) on the trailing edge $r / r_{0, \mathrm{CC}}$ $>1$, with a repeat of parallel and perpendicular regions that correspond to the oscillations in $g_{\mathrm{CC}}$. The comparison with the crystal structures is therefore misleading: The closest neighbors in the liquid are oriented preferentially parallel and not perpendicular for both $\mathrm{CO}_{2}$ and $\mathrm{CS}_{2}$.

\section{CONCLUSION}

In conclusion we find that direct determination of partial structure factors via a $2 N+X$ experiment involving the $\mathrm{C}$ isotope contrast gives unconvincing results even for the systems studied here, $\mathrm{CO}_{2}$ and $\mathrm{CS}_{2}$, that contain 33 at. \% $\mathrm{C}$. Instead we have developed a modeling approach for these systems that seeks the best representation of the three independent scattering data sets with a simulation. We explicitly restrict our search for models to rigid molecules that interact via 12-6-1 potentials and for which the $(p, \rho, T)$ $=(p, \rho, T)_{\exp }$. Even though the resulting partial pair distribution functions show distinct differences, the orientational correlations are similar with (always) preferentially parallel orientations for the closest neighbors. Based on these results, a comparison of the liquid structures with the crystals is misleading.

\section{ACKNOWLEDGMENTS}

We wish to acknowledge the help of P. Palleau (ILL) and R. Nowak (HASYLAB) during the experiments. Email discussion with D. Paschek (Univ. Dortmund) led to the use of rigid molecule models. SNS is managed by UT-Battelle, LLC, under Contract No. DE-AC05-00OR22725 for the U.S. Department of Energy. Contributions of J.M.S. to this research were conducted at the Center for Nanophase Materials Sciences, which is sponsored at Oak Ridge National Laboratory by the Division of Scientific User Facilities, U.S. Department of Energy.

\footnotetext{
${ }^{1}$ For example, A. I. Cooper, J. D. Londono, G. Wignall, J. B. McClain, E. T. Samulski, J. S. Lin, A. Dobrynin, M. Rubinstein, A. L. C. Burke, J. M. J. Fréchet, and J. M. DeSimone, Nature (London) 389, 368 (1997).

${ }^{2}$ U. Cubasch, G. A. Meehl, G. J. Boer, R. J. Stouffer, M. Dix, A. Noda, C. A. Senior, S. Raper, and K. S. Yap, 2001: Projections of future climate change, in Climate Change 2001: The Scientific Basis. Contribution of Working Group I to the Third Assessment Report of the Intergovernmental Panel on Climate Change, edited by J. T. Houghton, Y. Ding, D. J. Griggs, M. Noguer, P. van der Linden, X. Dai, K. Maskell, and C. I. Johnson (Cambridge University Press, New York, 2001).

${ }^{3}$ The Nobel Peace Prize 2007: http://nobelprize.org/nobel_prizes/peace/ laureates/2007/index.html.

${ }^{4}$ P. Cipriani, M. Nardone, F. P. Ricci, and M. A. Ricci, Mol. Phys. 99, 301 (2001).

${ }^{5}$ P. Jovari, Mol. Phys. 97, 1149 (1999).

${ }^{6}$ T. Morita, K. Nishikawa, M. Takematsu, H. Iida, and S. Furutaka, J. Phys. Chem. B 101, 7158 (1997).

${ }^{7}$ K. Suzuki and P. A. Egelstaff, Can. J. Phys. 81, 1389 (1974).

${ }^{8}$ B. M. Mognetti, M. Oettel, L. Yelash, P. Virnau, W. Paul, and K. Binder, Phys. Rev. E 77, 041506 (2008).

${ }^{9}$ Z. Zhang and Z. Duan, J. Chem. Phys. 122, 214507 (2005).

${ }^{10}$ A. E. Nasrabad and R. J. Laghaei, Chem. Phys. 125, 154505 (2006).
} 
${ }^{11}$ M. Saharay and S. Balasubramanian, J. Chem. Phys. 120, 9694 (2004).

${ }^{12}$ G. J. Ackland and S. J. Clark, Mol. Phys. 99, 855 (2001).

${ }^{13}$ B. R. Orton, Z. Naturforsch. 32, 863 (1977).

${ }^{14}$ S. I. Sandler and A. H. Narten, Mol. Phys. 32, 1543 (1976).

${ }^{15}$ D. L. Price, M.-L. Saboungi, and A. C. Barnes, Phys. Rev. Lett. 81, 3207 (1998).

${ }^{16}$ S. E. McLain, C. J. Benmore, S. E. Siewenie, J. Urquidi, and J. E. C. Turner, Angew. Chem., Int. Ed. 43, 1952 (2004).

${ }^{17}$ P. S. Salmon, R. A. Martin, P. E. Mason, and G. J. Cuello, Nature (London) 435, 75 (2005).

${ }^{18}$ A. C. Barnes, S. B. Lague, M. A. Hamilton, H. E. Fischer, A. N. Fitch, and E. Dooryhee, J. Phys.: Condens. Matter 10, L645 (1998).

${ }^{19}$ R. Ishii, S. Okazaki, I. Okada, M. Furusaka, N. Watanabe, M. Misawa, and T. Fukunaga, J. Chem. Phys. 105, 7011 (1996).

${ }^{20}$ T. Bausenwein, H. Bertagnolli, D. Gutwerk, K. Tödheide, and P. Chieux, Mol. Phys. 76, 127 (1992).

${ }^{21}$ For example, http://de.wikipedia.org/wiki/Eigenschaften_des_Wassers.

${ }^{22}$ B. Tomberli, C. J. Benmore, P. A. Egelstaff, J. Neuefeind, and V. Honkimaki, J. Phys.: Condens. Matter 12, 2597 (2000).

${ }^{23}$ P. A. Egelstaff, An Introduction to the Liquid State, 2nd ed. (Oxford University Press, Oxford, 1992).

${ }^{24}$ V. Iota, C. S. Yoo, J. H. Klepeis, Z. Jenei, W. Evans, and H. Cynn, Nature Mater. 6, 34 (2007).

${ }^{25}$ J. A. Montoya, R. Rousseau, M. Santoro, F. Gorelli, and S. Scandolo, Phys. Rev. Lett. 100, 163002 (2008).

${ }^{26}$ A. Simon and K. Peters, Acta Crystallogr., Sect. B: Struct. Crystallogr. Cryst. Chem. 36, 2750 (1980).

${ }^{27}$ N. C. Baenziger and W. I. Duax, J. Chem. Phys. 48, 2974 (1968).

${ }^{28}$ H. E. Fischer, J. Neuefeind, J. M. Simonson, R. Loidl, and H. Rauch, J. Phys.: Condens. Matter 20, 045221 (2008).
${ }^{29}$ R. L. McGreevy, J. Phys.: Condens. Matter 13, R877 (2001).

${ }^{30}$ A. K. Soper, Mol. Phys. 99, 1503 (2001).

${ }^{31}$ H. E. Fischer, G. J. Cuello, P. Palleau, D. Feltin, A. C. Barnes, Y. S. Badyal, and J. M. Simonson, Appl. Phys. A; Mater. Sci. Process. 74, S160 (2002).

${ }^{32}$ R. Bouchard, D. Hupfeld, T. Lippmann, J. Neuefeind, H.-B. Neumann, H. F. Poulsen, U. Rütt, T. Schmidt, J. R. Schneider, J. Süßenbach, and M. v. Zimmermann, J. Synchrotron Radiat. 5, 90 (1998).

${ }^{33}$ V. Honkimäki, (2007), http://www.esrf.eu/UsersAnd Science/ Experiments/MaterialsScience/ID15/.

${ }^{34}$ J. Neuefeind, H. E. Fischer, and W. Schröer, J. Phys.: Condens. Matter 12, 8765 (2000).

${ }^{35}$ O. I. Bashkin, K. V. Fedotov, V. M. Nefedova, G. V. Tissen, G. E. Ponyatovsky, A. Schiwek, and B. W. Holzapfel, Phys. Rev. B 68, 054401 (2003).

${ }^{36}$ W. Dmowski and T. Egami, J. Mater. Res. 22, 412 (2007).

${ }^{37}$ D. Paschek, A. Geiger, moscito 4, Department of Physical Chemistry University of Dortmund, 2002.

${ }^{38}$ L. Verlet, Phys. Rev. 159, 98 (1967).

${ }^{39}$ J.-P. Ryckaert, G. Ciccotti, and H. J. C. Berendsen, J. Comput. Phys. 23, 327 (1977).

${ }^{40}$ U. Essmann, L. Perera, M. L. Berkowitz, T. A. Darden, H. Lee, and L. G. Pedersen, J. Chem. Phys. 103, 8577 (1995).

${ }^{41}$ J. G. Harris and K. H. Yung, J. Phys. Chem. 99, 12021 (1995).

${ }^{42}$ S. B. Zhu, J. Lee, and G. W. Robinson, Mol. Phys. 65, 65 (1988).

${ }^{43}$ G. Ciccotti, M. Ferrario, and J.-P. Ryckaert, Mol. Phys. 47, 1253 (1982).

${ }^{44}$ L. Temleitner and L. Pusztai, J. Phys.: Condens. Matter 19, 335203 (2007).

${ }^{45}$ B. van Laar and W. B. Yelon, J. Appl. Crystallogr. 17, 47 (1984). 